\title{
Two-Spinon and Orbital Excitations of the Spin-Peierls System TiOCI
}

\author{
S. Glawion, ${ }^{1}$ J. Heidler, ${ }^{1}$ M. W. Haverkort, ${ }^{2}$ L. C. Duda, ${ }^{3}$ T. Schmitt, ${ }^{4}$ V. N. Strocov, ${ }^{4}$ C. Monney, ${ }^{4}$ K. J. Zhou, ${ }^{4}$ \\ A. Ruff, ${ }^{1}$ M. Sing, ${ }^{1}$ and R. Claessen ${ }^{1}$ \\ ${ }^{1}$ Experimentelle Physik 4, Universität Würzburg, 97074 Würzburg, Germany \\ ${ }^{2}$ Max Planck Institute for Solid State Research, 70569 Stuttgart, Germany \\ ${ }^{3}$ Department of Physics and Materials Science, Uppsala Universitet, 75121 Uppsala, Sweden \\ ${ }^{4}$ Swiss Light Source, Paul Scherrer Institut, 5232 Villigen PSI, Switzerland
}

(Received 8 November 2010; revised manuscript received 4 May 2011; published 1 September 2011; corrected 6 September 2011)

\begin{abstract}
We combine high-resolution resonant inelastic $\mathrm{x}$-ray scattering with cluster calculations utilizing a recently derived effective magnetic scattering operator to analyze the polarization, excitation energy, and momentum-dependent excitation spectrum of the low-dimensional quantum magnet $\mathrm{TiOCl}$ in the range expected for orbital and magnetic excitations $(0-2.5 \mathrm{eV})$. Ti $3 d$ orbital excitations yield complete information on the temperature-dependent crystal-field splitting. In the spin-Peierls phase we observe a dispersive two-spinon excitation and estimate the inter- and intradimer magnetic exchange coupling from a comparison to cluster calculations.
\end{abstract}

DOI: 10.1103/PhysRevLett.107.107402

PACS numbers: 78.70.Ck, 71.27.+a, 71.70.Ch, 78.70.Dm

Understanding the interplay of lattice, charge, orbital, and spin degrees of freedom in strongly correlated electron systems involving frustration remains one of the great tasks in modern solid state physics. The role of electronic and magnetic excitations for, e.g., the Mott metal-insulator transition is a key question in this respect [1]. Resonant inelastic x-ray scattering (RIXS) has been established recently as a powerful experimental technique to study these phenomena in complex solids [2-13], promoted by considerably improved energy resolution and detector sensitivity $[14,15]$. While previously inelastic neutron scattering was the method of choice for magnetic excitations, RIXS nowadays is established to give complementary information already from much smaller sample volumes $\left(<0.1 \mathrm{~mm}^{3}\right)$. This is due to the much larger scattering cross section of $\mathrm{x}$ rays compared to neutrons. Performing RIXS with photon energies close to the $L$ absorption edge corresponds to processes of the form $2 p^{6} 3 d^{n} \rightarrow$ $2 p^{5} 3 d^{n+1} \rightarrow 2 p^{6} 3 d^{n *}$. The intermediate state is the final state of x-ray absorption spectroscopy (XAS), and the RIXS final state can be an excited state (indicated by the asterisk) with losses due to coupling to all kinds of excitations. For $n=1$ the initial and final state can be described by single-particle wave functions, in contrast to the intermediate state. Thus, although it is possible to extract the crystal-field (CF) splitting directly from orbital $(d d)$ excitations, a many-body approach is necessary to simulate RIXS spectra.

In this Letter, we analyze the temperature, excitation energy, polarization, and momentum dependence of the magnetic and orbital excitations of the quasi-onedimensional spin-Peierls system $\mathrm{TiOCl}$ measured by high-resolution RIXS. The data allow us to extract precise values for the CF splitting in the room temperature (RT) as well as the spin-Peierls phase. The polarization dependence of the CF levels is in very good agreement with cluster calculations based on density-functional theory within the local-density approximation. From comparison to cluster calculations for a dimerized spin chain we identify a low-energy feature as a two-spinon excitation, amplifying the quasi-one-dimensional nature of TiOCl. The predictive power of a recently derived effective scattering operator for magnetic excitations in RIXS is evidenced for the first time by simulating the intensity of this excitation for several different momenta in relation to the $d-d$ excitation strength. Furthermore, by including the dimerization into our theory such that the spin gap found in other experiments [16] is reproduced we can infer the ratio of the inter- and intradimer magnetic exchange coupling.

$\mathrm{TiOCl}$ is a layered system of buckled Ti-O bilayers sandwiched by $\mathrm{Cl}$ ions. Such layers are stacked along the $c$ axis with only weak van der Waals forces present in between, allowing for easy cleavage. The structure thus effectively confines electronic and magnetic interactions to the $a b$ plane. In this plane, Ti ions form a staggered triangular lattice which introduces magnetic frustration. Locally, each $\mathrm{Ti}$ ion is coordinated by four $\mathrm{O}$ and two $\mathrm{Cl}$ ions which form a strongly distorted octahedron with a local $C_{2 v}$ point-group symmetry in the orthorhombic crystal structure. This results in a completely lifted degeneracy of the $3 d$ orbitals, i.e., five energetically well separated CF levels, and thus a quenching of the orbital degrees of freedom [17]. The Ti ions are in a $3 d^{1}$ configuration and the overlap of occupied $d_{x^{2}-y^{2}}$ orbitals along $b$ provides a onedimensional (1D) hopping path, leading to (quasi-)1D behavior in several aspects [18]. For example, below $T_{c 1}=67 \mathrm{~K}$ the system shows a dimerization of $\mathrm{Ti}$ ions along $b$ into $S=0$ singlets, i.e., a spin-Peierls phase, with a huge spin gap of approximately $430 \mathrm{~K}=37 \mathrm{meV}$ [16]. 
At higher temperatures the magnetic susceptibility corresponds to that of a 1D Heisenberg chain, with a nearestneighbor exchange coupling $J \approx 660 \mathrm{~K}=57 \mathrm{meV}$ [19]. The spin-Peierls ground state below $67 \mathrm{~K}$ is of considerable interest since it is reached via an incommensurately modulated intermediate phase below $T_{c 2}=91 \mathrm{~K}$ [20]. Frustrated interchain interactions are widely accepted as the driving force for this unconventional behavior (cf. Ref. [21] and references therein).

Samples were grown by a chemical vapor transport method [22], with a typical size of $(1 \times 2 \times 0.1) \mathrm{mm}^{3}$. Measurements were conducted at the SAXES end station $[14,15]$ of the ADRESS beam line (spot size $52 \times 4 \mu \mathrm{m}^{2}$ ) at the Swiss Light Source (Paul Scherrer Institut, Switzerland) using soft $x$-ray RIXS with an energy resolution of $80 \mathrm{meV}$ or better, as determined from the full width at half maximum of the elastic line of a carbon powder sample at the relevant excitation energies. As shown in the insets of Fig. 1 the end station allows different angles $\phi$ between the incoming and outgoing beam, as well as a rotation of the sample around the polar axis for the momentum-dependent measurements. For $\phi=90^{\circ}$ and $\pi$-polarized (i.e., within the scattering plane) incoming light the polarization of the outgoing beam must necessarily be rotated. For $\sigma$-polarized (i.e., perpendicular to the scattering plane) incoming light this need not be the case. This leads to well-defined polarization effects even without detecting the polarization of the outgoing photons. We used $\phi=90^{\circ}$ and a grazing incidence angle $\theta=20^{\circ}$ for all RIXS measurements except those to investigate the momentum dependence. In the latter case, only the

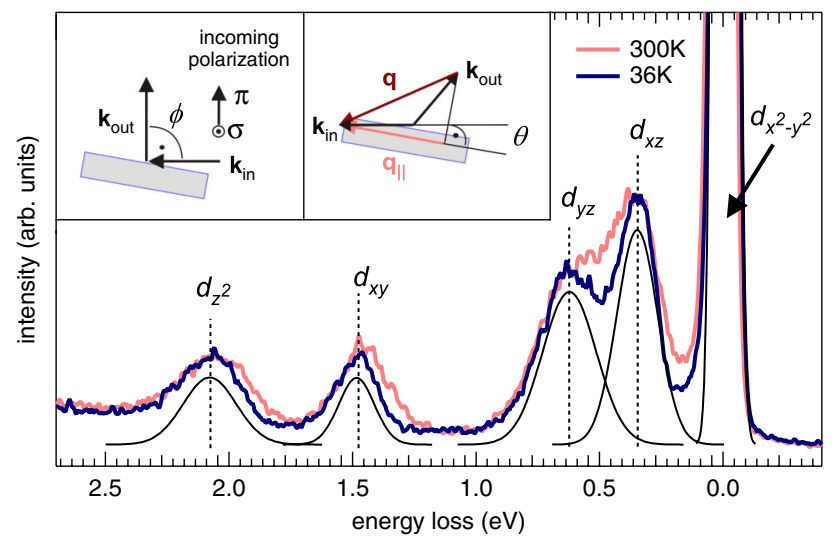

FIG. 1 (color online). RIXS spectra at $T=300 \mathrm{~K}$ (light red) and $T=36 \mathrm{~K}$ (dark blue) $(h \nu=454.2 \mathrm{eV}, \sigma$ polarization, $a c$ scattering; see text). Black solid curves are Gaussian fits to the low- $T$ data. Also, the orbital character of the $\mathrm{CF}$ excitations is indicated. Insets: Experimental RIXS geometry. Left: $\phi=90^{\circ}$ geometry to define $\sigma$ (out-of-plane) and $\pi$ (in-plane) polarizations. Right: Definition of $\boldsymbol{q}$ (brown arrow) and $q_{\|}$(red arrow). $\boldsymbol{q}$ was fixed by the constant $\phi=50^{\circ}$ in our experiments, and $q_{\|}$was varied by rotating the sample around the polar axis, i.e., changing $\theta$. projection of the momentum transfer $\boldsymbol{q}=\boldsymbol{k}_{\text {in }}-\boldsymbol{k}_{\text {out }}$ on the sample surface $\left(q_{\|}\right.$, see right inset of Fig. 1) is of importance due to the quasi-1D nature of TiOCl. Because of the bulk sensitivity of RIXS governed by the attenuation length of the $\mathrm{x}$ rays ( $>100 \AA$ ), it was sufficient to cleave samples in air using Scotch tape and then transfer them into the vacuum $\left(<10^{-9}\right.$ mbar $)$. Acquisition times per spectrum were in the range 90-200 min. To simulate the $d d$ excitations we used a (paramagnetic) $\mathrm{TiO}_{4} \mathrm{Cl}_{2}$ cluster within multiplet ligand-field theory, a powerful and wellestablished many-body approach to x-ray absorption and emission processes, especially in strongly correlated electron systems [23]. Hopping parameters and Slater integrals were obtained from local-density approximation calculations followed by a downfolding procedure [24,25]. The cluster calculations account for the CF splitting as well as the covalence between the Ti $3 d$ and $\mathrm{O} 2 p$ orbitals. The magnetic excitations have been calculated from two separate clusters utilizing a recently derived effective scattering operator for RIXS of collective magnetic excitations [10]. The X-ray absorption spectra that enter within this theory as the energy-dependent resonant enhancement prefactors were obtained from the same cluster as the $d d$ excitations. For the two-spinon excitations we used a cluster of 28 sites for a 1D dimerized Heisenberg chain with nearestneighbor exchange.

Figure 1 shows typical RIXS spectra at $T=300 \mathrm{~K}$ (red curve) and at $T=36 \mathrm{~K}$ (blue curve), i.e., in the spinPeierls phase, taken at a photon energy $h \nu=454.2 \mathrm{eV}$ in $\sigma$ polarization with $a c$ being the scattering plane ("ac scattering"). In both cases, five peaks are observable, corresponding to the on-site $d d$ excitations. For the first time, all CF excitations of $\mathrm{TiOCl}$ are mapped directly in one experiment, as in other (optical) probes some of them are forbidden due to dipole selection rules [26]. Besides the elastic line at zero energy loss four peaks appear at $0.34,0.62,1.48$, and $2.08 \mathrm{eV}$ [27], as determined from fitting Gaussian peaks to the low- $T$ data. At $300 \mathrm{~K}$, they lie at $0.36,0.59,1.45$, and $2.05 \mathrm{eV}$, respectively, due to $T$-dependent changes in the distortion of the $\mathrm{TiO}_{4} \mathrm{Cl}_{2}$ octahedra predicted by cluster calculations [26].

Figure 2(a) shows RIXS spectra (solid red curves) at different photon energies across the $\mathrm{Ti} L$ edge $(T=300 \mathrm{~K})$. Also shown are results from cluster calculations (dashed blue curves), including $60 \mathrm{meV}$ resolution and $200 \mathrm{meV}$ (final-state) lifetime broadening as inferred from experiment. The elastic line has zero weight in the simulations (except for Bragg reflections). This is expected for the perfectly ordered, infinite crystal assumed in our calculations. In experiment, surface reflectivity and scattering off crystal imperfections always produce a (quasi-) elastic peak. The overall agreement between theory and experiment is very good, and aside from sample quality the remaining discrepancies might be connected to uncertainties concerning the exact excitation energy. This is 

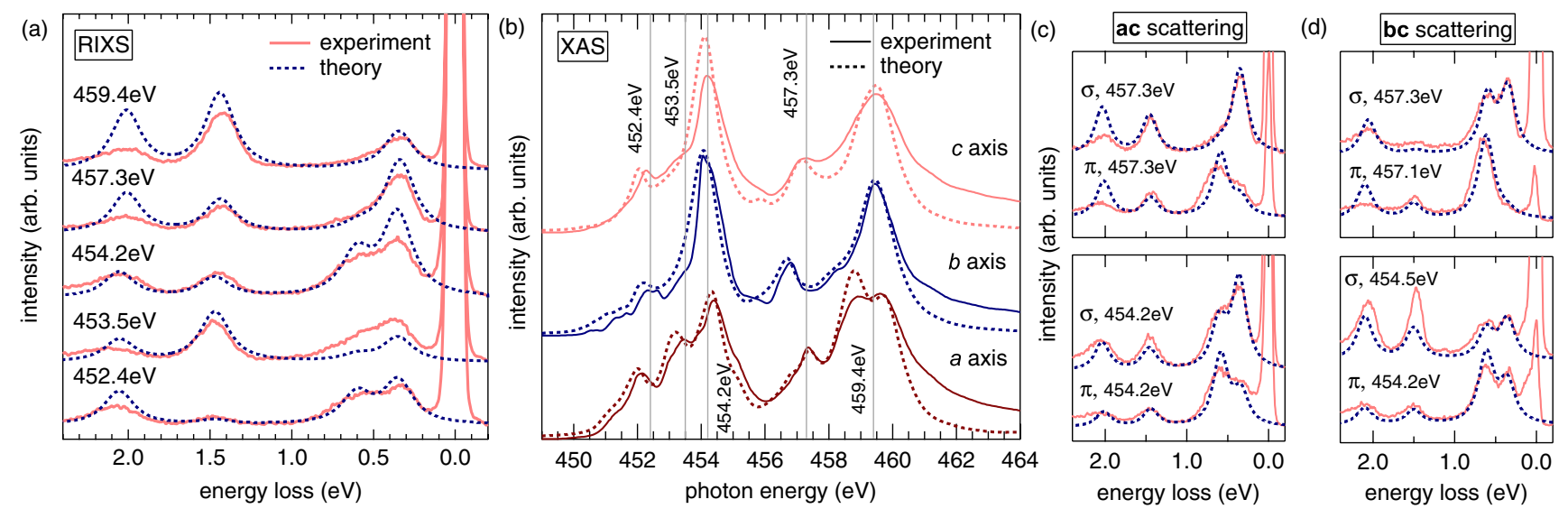

FIG. 2 (color online). (a) Excitation-energy dependence of RIXS spectra at $T=300 \mathrm{~K}$ in $\sigma$ polarization and $a c$ scattering. Corresponding excitation energies are indicated. (b) Ti $L$ edge XAS spectra with main polarizations along the different crystal axes both measured (solid curves) and calculated (dashed curves) for the geometry of panel (a). Photon energies from (a) are marked by vertical lines. (c),(d) $\sigma$ - and $\pi$-polarized RIXS spectra for different photon energies in $a c$ (c) and $b c$ (d) scattering from experiments (solid curves) and multiplet calculations (broken curves).

illustrated by Fig. 2(b) which shows absorption spectra acquired in total electron yield, with the (main) polarization [28] and the used photon energies indicated. The XAS consists of a complicated multiplet structure, and being off in photon energy by a few meV can lead to largely different intensities for the involved dipole transitions. Since XAS is the intermediate state of RIXS the agreement between simulated and measured RIXS spectra cannot be better than for the absorption spectra. Note that the intermediate state broadening for the RIXS spectra is equivalent to the broadening used for the calculation of the XAS spectra. The core-hole broadening varies between $200 \mathrm{meV}$ for the low-energy states $(450 \mathrm{eV})$ to $600 \mathrm{meV}$ for the high energy states $(465 \mathrm{eV})$. The same also holds for the spectra shown in Figs. 2(c) and 2(d), where the effects of different polarization and scattering geometries are compared at two excitation energies. Again, although some discrepancies remain there is notable agreement between theory and experiment. Note that in the experimental data the elastic line is strongly suppressed in $\pi$ polarization $[29,30]$.

A feature barely visible in the experimental spectra shown up to now is a low-energy excitation (LEE) centered at roughly $0.1 \mathrm{eV}$, which is not captured by the paramagnetic cluster calculations. Figure 3(a) shows momentumdependent RIXS spectra within an energy window containing only the two lowest $d d$ excitations and the elastic line measured at $T=15 \mathrm{~K}$ for different $q_{\|}$along $b$ ( $\pi$ polarization, $b c$ scattering). Since for some samples the LEE feature started to disappear after 5 min of beam exposure, the sample spot was changed every 5 min by $10 \mu \mathrm{m}$. Note, however, that also for such samples the behavior of the LEE completely agrees with the data shown here, taken on samples, which did not show any degradation of the spectra up to $20 \mathrm{~min}$ of irradiation. Simulated spectra in Fig. 3(a) consist of a superposition of three parts. In addition to $d d$ excitations with a reduced lifetime broadening of $150 \mathrm{meV}$ due to the low measuring temperature, spinon calculations for the LEE based on the above-mentioned effective operator [10], and a Gaussian to mimic the elastic peak are incorporated. Since at $h \nu=460 \mathrm{eV}$ one has $q \approx 0.413 \AA^{-1}$ and the Brillouin zone (BZ) boundary along the 1D direction (the $Y$ point) lies at $0.94 \AA^{-1}$ we could cover approximately $1 / 3$ of the path $\Gamma Y$ with $q_{\|}$.
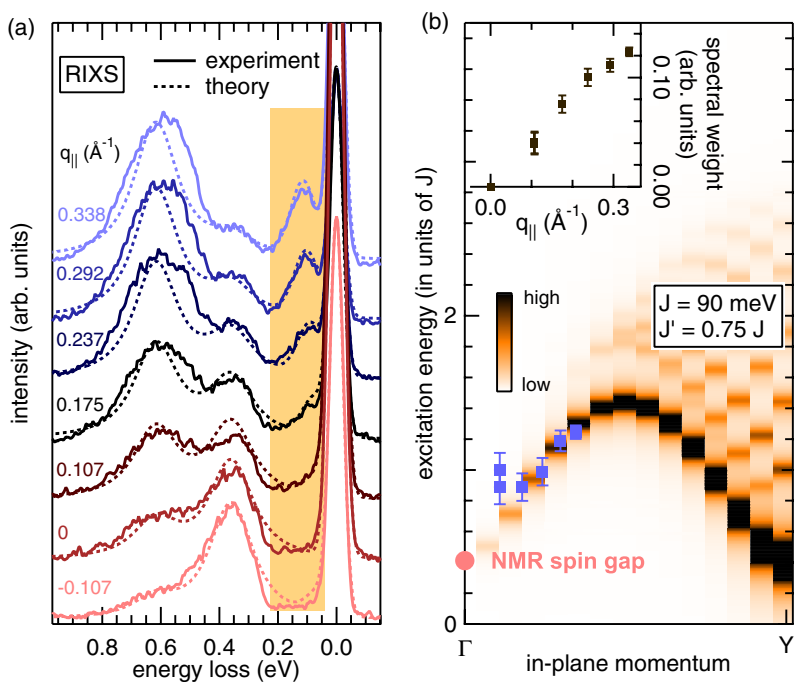

FIG. 3 (color online). (a) RIXS spectra taken at $h \nu=$ $456.8 \mathrm{eV}$ in $\pi$ polarization and $b c$ scattering $(T=15 \mathrm{~K})$, for $q_{\|}$corresponding to (from top to bottom) $36 \%, 31 \%, 25 \%, 19 \%$, $11 \%, 0 \%$, and $-11 \%$ of the $\Gamma Y$ path in the BZ. The LEE is highlighted by the shaded area. (b) Two-spinon dynamical spin structure factor for a 28 site dimerized Heisenberg model. The dispersion of the LEE is indicated, obtained from fitting the data in panel (a) (blue square markers). At $q_{\|}=0$ the spin gap extracted from NMR [16] is indicated. Inset: LEE spectral weight (i.e., experimental peak areas). 
The LEE is most pronounced in the spectrum with the highest $q_{\|}=0.338 \AA^{-1}$ [top of Fig. 3(a)]. Towards the $\Gamma$ point it rapidly loses intensity, and a dispersion from $\sim 110 \mathrm{meV}$ to $\sim 80 \mathrm{meV}$ is observed. Its nature becomes evident from looking at the two-spinon dynamical structure factor for a 1D chain [31,32]. It describes a continuum of excited states with two sinelike (quasi-) boundaries $\omega_{L}(q)$ and $\omega_{U}(q)$, and its spectral weight $\int_{\omega_{L}(q)}^{\omega_{U}(q)} S(q, \omega) d \omega$ goes linearly to zero towards the zone center. To take dimerization into account we calculated the dynamical structure factor for a 28 site Heisenberg spin chain with intra- and interdimer nearest-neighbor interactions $J$ and $J^{\prime}$, respectively. As can be seen in Fig. 3(b) a spin gap opens at the zone center and the zone boundary. The intensity, however, still goes linearly to zero towards the zone center as for the undimerized chain. Note that in the experiment the in-plane momentum and the polarization are changed simultaneously between different spectra in Fig. 3(a) due to the experimental setup. This has been accounted for in the calculations, but the dependence of the spectral weight on the polarization for this specific case is only of the order of $10 \%$. Consequently, there is good agreement for the intensity ratios of both the spin and the orbital excitations. The experimental dispersion (blue square markers) and the spin gap from NMR (red dot, $37 \mathrm{meV}$ ) [16] are best reproduced for exchange couplings of $J=90 \mathrm{meV}$ and $J^{\prime}=0.75 J$, respectively, i.e., their mean value is in reasonable agreement with the value of $57 \mathrm{meV}$ for the undimerized chain. The inset shows that the experimental LEE weight goes linearly to zero, evidencing its two-spinon character. An analogous behavior of magnetic features in RIXS has previously been observed, e.g., on $\mathrm{La}_{2} \mathrm{CuO}_{4}$ [12], but $\mathrm{TiOCl}$ is the first noncuprate in which such an excitation could be identified.

In principle, $d d$ excitations might also be dispersive. The orbital exchange energy is of the same order as the magnetic exchange and smaller than their lifetime broadening. Since in RIXS the probability for flipping an orbital or a spin is roughly the same the experimental excitations likely involve coupled orbital and magnon excitations, eventually with additional damping from phonons. Looking back at Fig. 3(a) one notices that at large $q_{\|}$, e.g., the $d_{y z}$ excitation is significantly broader than the local cluster calculation. A detailed analysis of this observation, however, is out of the scope of the present study.

In conclusion, by overcoming the selection-rule limitations of optical spectroscopy using RIXS we have directly obtained reliable values for the $\mathrm{CF}$ splitting from the orbital $(d d)$ excitations of TiOCl. Their temperature, excitation energy, and polarization dependence is in agreement with results from cluster calculations. Evaluating the momentum-dependent behavior of a low-energy peak observed in the spin-Peierls phase on the energy scale of the magnetic exchange coupling $J$, we can identify this peak as a two-spinon excitation. Using a new effective operator for magnetic excitations in RIXS in combination with finite size cluster calculations, its dispersion and intensity is accounted for. Moreover, we find robust values for the inter- and intradimer exchange coupling.

We acknowledge fruitful discussions with F.F. Assaad and J. Schlappa. This work was supported by the German Science Foundation (DFG) via Grants No. CL-124/6-1 and No. CL-124/6-2, the Swedish Research Council (VR), and the Göran Gustafsson Foundation (GGS). This work was performed at the ADRESS beam line using the SAXES instrument jointly built by Paul Scherrer Institut, Switzerland and Politecnico di Milano, Italy.

[1] M. Imada et al., Rev. Mod. Phys. 70, 1039 (1998).

[2] S. G. Chiuzbăian et al., Phys. Rev. Lett. 95, 197402 (2005).

[3] Y.-J. Kim et al., Phys. Rev. B 76, 155116 (2007).

[4] C. Ulrich et al., Phys. Rev. B 77, 113102 (2008).

[5] G. Ghiringhelli et al., Phys. Rev. Lett. 102, 027401 (2009).

[6] L. Braicovich et al., Phys. Rev. Lett. 102, 167401 (2009).

[7] J. Schlappa et al., Phys. Rev. Lett. 103, 047401 (2009).

[8] C. Ulrich et al., Phys. Rev. Lett. 103, 107205 (2009).

[9] L. J. P. Ament et al., Phys. Rev. Lett. 103, 117003 (2009).

[10] M. W. Haverkort, Phys. Rev. Lett. 105, 167404 (2010).

[11] L. Braicovich et al., Phys. Rev. Lett. 104, 077002 (2010).

[12] L. Braicovich et al., Phys. Rev. B 81, 174533 (2010).

[13] M. Guarise et al., Phys. Rev. Lett. 105, 157006 (2010).

[14] G. Ghiringhelli et al., Rev. Sci. Instrum. 77, 113108 (2006).

[15] V. N. Strocov et al., J. Synchrotron Radiat. 17, 631 (2010).

[16] T. Imai et al., arXiv:cond-mat/0301425.

[17] T. Saha-Dasgupta et al., Europhys. Lett. 67, 63 (2004).

[18] M. Hoinkis et al., Phys. Rev. B 72, 125127 (2005).

[19] A. Seidel et al., Phys. Rev. B 67, 020405(R) (2003).

[20] A. Krimmel et al., Phys. Rev. B 73, 172413 (2006).

[21] Y.-Z. Zhang, H. O. Jeschke, and R. Valenti, Phys. Rev. B 78, 205104 (2008).

[22] H. Schäfer et al., Z. Anorg. Allg. Chem. 295, 268 (1958).

[23] F. M. F. de Groot and A. Kotani, Core Level Spectroscopy of Solids (Taylor \& Francis, London, 2008).

[24] M. W. Haverkort et al. (to be published).

[25] O. K. Andersen and T. Saha-Dasgupta, Phys. Rev. B 62, R16219 (2000).

[26] R. Rückamp et al., New J. Phys. 7, 144 (2005).

[27] Error estimated as $\pm 0.01 \mathrm{eV}$.

[28] Because of the experimental geometry all spectra have non-negligible admixtures from other polarizations.

[29] M. van Veenendaal, Phys. Rev. Lett. 96, 117404 (2006).

[30] G. Ghiringhelli et al., Phys. Rev. Lett. 92, 117406 (2004).

[31] G. Müller et al., Phys. Rev. B 24, 1429 (1981).

[32] M. Karbach et al., Phys. Rev. B 55, 12510 (1997). 